\title{
Two-Phase Gas-Liquid Flow in Pipes with Different Orientations
}

\author{
Afshin J. Ghajar \\ Regents Professor and John Brammer Endowed Professor \\ School of Mechanical and Aerospace Engineering \\ Oklahoma State University, Stillwater, Oklahoma, USA \\ afshin.ghajar@okstate.edu
}

The phenomenon of gas-liquid two-phase flow in inclined systems, although not as common as horizontal or vertical flow, is of great practical significance in several applications such as undulating oil-gas flow lines, chemical process engineering, and inclined flow paths in steam condensers and generators. In these practical applications, accurate determination of two-phase flow variables such as void fraction, pressure drop and heat transfer is of great importance for system sizing and optimization. It is well established fact that these parameters are very sensitive to the spatial and morphological variations of the two-phase flow structure. The two-phase flow structure commonly termed as flow pattern, depends on the interaction and balance between the buoyancy-inertiagravity forces which in turn are a function of pipe inclination. Therefore, a correct understanding of the effect of change in pipe inclination on the two-phase flow structure is needed and its effect on the thermofluidics of twophase flow needs to be understood. Thus, the scope of this lecture is to present an overview of the pipe inclination effects of the gas-liquid two-phase flow phenomenon.

The pipe inclination effects on non-boiling two-phase flow are studied through the data available in the literature and extensive experiments carried out in the Two-Phase Flow Laboratory at Oklahoma State University. These experiments are carried out using air-water as fluid combination. The experimental setup is unique as it can be inclined from horizontal to vertical in both upward and downward inclinations. The setup is capable of flow visualization and simultaneous measurements of void fraction, pressure drop, and heat transfer. The experimental data show the flow pattern and pipe inclination dependency of all two-phase flow variables. 